\title{
Lipid Profile Abnormalities in Bangladeshi Type 2 Diabetic Patients Attending a Tertiary Care Hospital: A Cross-Sectional Study
}

\author{
Afsana Begum ${ }^{1 *}$, SM Rezaul Irfan²
}

\begin{abstract}
:
Diabetes Mellitus is one of the leading non-communicable diseases all over the world including Bangladesh. Diabetes is characterized by chronic hyperglycemia and disturbances of carbohydrate, lipid and protein metabolism. Impaired lipid profile is commonly present in type 2 diabetes. We aimed to research serum lipid profile abnormalities hypothesizing that early detection and treatment of lipid abnormalitiescan minimize the risk for atherogenic cardiovascular disorder and cerebrovascular accident in patients with type 2 diabetes mellitus. This observational cross sectional study was carried out in the department of Biochemistry, Bangladesh Institute of Research \& Rehabilitation in Diabetes, Endocrine and Metabolic Disorders (BIRDEM).A total 105patients with T2DM of age within the range of 30-45 years were selected \& their Fasting blood glucose (FBG), total cholesterol (TC), high density lipoprotein $(H D L)$, low density lipoprotein $(L D L)$, triglyceride $(T G)$ and glycatedhaemoglobin (HbAlc) levels were evaluated. Significantly higher mean serum levels of TC, TG and LDL and significantly lower mean serum levels of $H D L$ were noted in patients with diabetes. Significant correlations were observed between HbAlc value and serum levels of TC, TG and HDL $(p<0.05)$ but no correlation of $H b A_{1} c$ value withlow density lipoprotein in diabetes patient.The study showed widespread lipid abnormalities in the course of diabetes triggered dyslipidemia as hypercholesterolemia, hypertriglyceridemia, elevated LDL and decreased HDL.
\end{abstract}

Keywords: Total cholesterol (TC), Triglyceride (TG), High density lipoprotein cholesterol(HDL-C), Low density lipoprotein cholesterol(LDL-C), Dyslipidemia, Type 2 diabetes.

\section{Background:}

Diabetes mellitus (DM) is a heterogeneous condition reflecting different metabolic disorder accompanied by a variety of complications. This is characterized by hyperglycemia due to absolute or relative deficiency of insulin ${ }^{1}$.Lack of insulin whether absolute or relative affects the metabolism of carbohydrate, protein, fat, water and electrolytes $^{2}$. Insulin affects many sites of mammalian lipid metabolism. It stimulates synthesis of fatty acid in liver, adipose tissue and in the intestine. The insulin has also been reported to increase the cholesterol synthesis. The activity of lipoprotein lipase in white adipose tissue is also increased ${ }^{3}$.As early as in 1988, it was described a multifactorial metabolic abnormality consisting of insulin resistance with compensatory hyperinsulinaemia, type 2 diabetes mellitus (T2DM), essential hypertension and hypercholesterolaemia ${ }^{4,5}$. Today, however, the World Health Organization (WHO) and

1. Lecturer, Department of Biochemistry, Mugda Medical College, Mugda, Dhaka

2. Department of Cardiology, BIRDEM General Hospital, Dhaka, Bangladesh

\section{*Corresponding Author:}

Dr. Afsana Begum

MPhil (Medical Biochemistry)

Lecturer, Department of Biochemistry

Mugda Medical College, Mugda, Dhaka

Cell No: +88 01710919905

Email: afsana42@yahoo.com
International Diabetes Federation (IDF) use the term 'Metabolic Syndrome' to describe this clustering of conditions ${ }^{6}$. The term diabetic dyslipidemia comprises a triad of raised triglycerides, reduced high density lipoprotein (HDL) and excess of small, dense low density lipoprotein (LDL) particles. The lipid abnormalities are prevalent in diabetes mellitus because insulin resistance or deficiency affects key enzymes and pathways in lipid metabolism ${ }^{7}$. Micro-vascular and macro-vascular complications, including cardiovascular disease (CVD), retinopathy, nephropathy, and neuropathy, occur due to chronic uncontrolled hyperglycemia in diabetics ${ }^{8,9}$.It has been proposed that the composition of lipid particles in diabetic dyslipidemia is more atherogenic than other types of dyslipidemia ${ }^{10}$. The causal association between atherosclerosis and dyslipidemia is well established. In diabetes the associated hyperglycemia, obesity and insulin changes highly accelerate the progression to atherosclerosis ${ }^{11,12}$.In the present study, we aimed to research serum lipid profile abnormalities hypothesizing that early detection and treatment of lipid abnormalities can minimize the risk for atherogenic cardiovascular disorder and cerebrovascular accident in patients with type 2 diabetes mellitus.

\section{Materials and Methods:}

The cross-sectional study sample included Bangladeshi participants diagnosed with T2DM. The study was done from January 2017 to June 2017 in the outpatient departments of BIRDEM and participating 105 subjects with T2DM were randomly selected and recruited in the study. Ethical approval 
of the study was from the Ethical Review Committee of the Bangladesh Diabetic Association (BADAS). Informed written consent was obtained from each of the participants after explaining the objective of the study. Data were collected including demographic characteristics and clinical history by utilizing a pre-designed questionnaire and were collected by direct interview from participants. Relevant physical examinations were performed on all participants. With all aseptic precaution about $5 \mathrm{ml}$ blood was collected from all of the subjects. Serum was separated after centrifuging at 3000 rpm for 10 minutes. The sera obtained and were separated. Blood glucose - both fasting and OGTT, $\mathrm{HbA}_{1 \mathrm{C}}, \mathrm{CBC}$, Total cholesterol, Triglyceride, High density lipoprotein (HDL) cholesterol, Low density lipoprotein (LDL) cholesterol level were obtained. $\mathrm{HbA}_{1 \mathrm{C}}$ was estimated in whole blood by a BIO-RAD variant which was modified HPLC method. All the biochemical tests were done in the Department of Cell and Molecular Biology, BIRDEM following standard methods and procedures.

Data were analyzed using the Statistical Package for the Social Sciences version 16.0 (SPSS, Chicago, IL, USA). Normal continuous variables were presented as mean \pm standard deviation. Test of significance was calculated by unpaired student's t test. Correlation studies (Pearson's correlation) were performed between the variables of blood glucose and serum lipid profile. Significance was set at $\mathrm{p}<0.05$.

\section{Results:}

We evaluated clinical and laboratory findings in 105 patients with T2DM. Of the total number of subjects, $41 \%$ (43) were females, and $59 \%$ (62) of the subjects were males (Table 1).

Clinical variables including Body mass index (BMI), Systolic blood pressure (SBP) and Diastolic blood pressure (DBP) was observed in the study subjects(Table 2). However, a significant difference was found as compared to normal range for related serum lipids of diabetics $(\mathrm{p}<0.001)$ (Table-3).

In Pearson's correlation studies, showed significant positive correlation of $\mathrm{HbA}_{1} \mathrm{c}$ with TC, TG and HDL $(\mathrm{p}<0.05)$ (Table-4).

Table-1: Gender distribution and age of patients with type 2 diabetes

\begin{tabular}{lcc}
\hline Gender & No. of patients (\%) & Age (year) \\
\hline Female & $43(40.95 \%)$ & $37.51 \pm 5.082$ \\
Male & $62(59.04 \%)$ & $39.39 \pm 4.829$ \\
Total & $105(100.00 \%)$ & $38.62 \pm 4.997$ \\
\hline
\end{tabular}

Data were expressed as numbers (n) and percentages (\%)

Table-2: Clinical variables of the study subjects

\begin{tabular}{lcc}
\hline Clinical variables & Female & Male \\
\hline Mean $( \pm$ SD) BMI & $24.5 \pm 2.9$ & $23.9 \pm 2.9$ \\
Mean $( \pm$ SD) SBP & $130.8 \pm 45.9$ & $146.2 \pm 51.6$ \\
Mean $( \pm$ SD) DBP & $83.4 \pm 19.8$ & $87.4 \pm 19.8$ \\
\hline
\end{tabular}

(Results are expressed as mean $\pm S D ; S D$, Standard deviation; BMI, Body mass index; SBP, Systolic blood pressure; DBP, Diastolic blood pressure; Blood pressure in $\mathrm{mm} \mathrm{Hg.)}$

Table-3: Biochemical variables of the study subjects

\begin{tabular}{lcccc}
\hline Biochemical Variables & Normal range & Female & Male & P value \\
\hline Mean $( \pm$ SD) FBG $(\mathbf{m m o l} / \mathbf{L})$ & $3.5-6.1$ & $11.84 \pm 3.5$ & $12.75 \pm 3.9$ & $.001 *$ \\
Mean $( \pm$ SD) HbA $\mathbf{1 C}(\mathbf{m})$ & $4-6$ & $9.98 \pm 1.65$ & $10.40 \pm 2.2$ & $.001 *$ \\
Mean $( \pm$ SD) TG $(\mathbf{m g} / \mathbf{d l})$ & $35-116$ & $196.74 \pm 128.92$ & $213.61 \pm 145.32$ & $.001 *$ \\
Mean $( \pm$ SD) TC $(\mathbf{m g} / \mathbf{d l})$ & $150-220$ & $204.63 \pm 43.38$ & $215.16 \pm 46.47$ & $.001 *$ \\
Mean $( \pm$ SD) HDL $(\mathbf{m g} / \mathbf{d l})$ & $>40$ & $38.5 \pm 8.85$ & $34.66 \pm 8.6$ & $.001 *$ \\
Mean $( \pm$ SD) LDL $(\mathbf{m g} / \mathbf{d l})$ & $80-130$ & $143.28 \pm 41.72$ & $133.77 \pm 39.71$ & $.001 *$ \\
\hline
\end{tabular}

(Results expressed as mean $\pm S D$; SD, Standard deviation; FBG, Fasting blood glucose; TG, Triacylglyceride; TC, Total cholesterol; HDL, High density lipoprotein cholesterol; LDL, Low density lipoprotein cholesterol.)

Table -4: Correlation of $\mathrm{HbA}_{1} \mathrm{c}$ with lipids in study subjects

\begin{tabular}{lccc}
\hline Glycated hemoglobin & Lipid Profile & $\mathbf{r}$ & $\mathbf{p}$ \\
\hline HbA $_{1} \mathbf{c}$ & TC & 0.047 & 0.003 \\
& HDL & 0.686 & 0.003 \\
& LDL & 0.186 & 0.058 \\
& TG & 0.767 & 0.001 \\
\hline
\end{tabular}

Pearson's correlation coefficient was performed for the analysis; $\mathrm{p}<0.05$ was considered as statistically significant; $\mathrm{TC}=\mathrm{Total}$ cholesterol, HDL $=$ High density lipoprotein, LDL $=$ Low density lipoprotein, TG $=$ Triglyceride. Significant correlation. 


\section{Discussion:}

Diabetes has become a major health problem in the world. The prevalence of diabetes and its adverse health effects is increasing more rapidly in South Asia than in any other large region of the world.

There were more males (59\%) than females (41\%) with T2DM in this study. The high proportion of males in this study may be due to the nature of population admitting to this hospital in that more of them seek medical attention than women under favour of having more responsive towards health awareness because most of them were employed.

In the present study, significantly higher mean serum levels of total cholesterol, triglycerides and LDL cholesterol were noted in patients with diabetes, which are well known risk factors for cardiovascular diseases among patients, when compared to the normal values (Table-3). This is in agreement with OnyemelukweandStafford, Bello- Sani et al., and Rashastudies ${ }^{13-15}$.This is attributed to insulin deficiency which results in faulty glucose utilization, causing hyperglycemia and mobilization of fatty acids from adipose tissue ${ }^{16}$. The fatty acids from adipose tissue are mobilized for energy purpose and excess fatty acids are accumulated in liver, which are converted to triglyceride ${ }^{16}$. LDL cholesterol in diabetes was found to be significantly increased which is consistent with the findings of Bello- Sani et al. ${ }^{14}$.In patients with diabetes, many studies have clearly established that complications are mainly due to chronic hyperglycemia that exerts its injurious to health effects through several mechanisms: dyslipidemia, platelet activation, and altered endothelial metabolism ${ }^{17,18,19}$. Both lipid profile and diabetes have been shown to be the important predictors for metabolic disturbances including dyslipidaemia, hypertension, cardiovascular diseases $^{20}$. Lipids play a vital role in the pathogenesis of diabetes mellitus. Dyslipidemia as a metabolic abnormality is frequently associated with diabetes mellitus. Abnormalities in lipid metabolism have been reported in patients with diabetes mellitus accompanied by the risk of cardiovascular arteriosclerosis ${ }^{21}$.In the present study, significant correlations were observed between serum levels of total cholesterol, triglycerides, HDL cholesterol and blood concentrations of $\mathrm{HbA}_{1} \mathrm{c}$ (Table -4). Results of the correlation studies suggest a clear association between hyperglycemia and appearance of dyslipidemia. Our findings were in line with a previous study suggesting that the level of total cholesterol is usually normal or near normal if glycaemic control is adequate, and worsening of control raises the level ${ }^{22}$. Therefore, improving glycaemic control might substantially reduce the risk of cardiovascular events in diabetic patients.

Our study clearly shows that lipid profiles are abnormal in diabetes mellitus. Realizing that most of the diabetics have a high probability of developing cardiovascular and cerebrovascular disease, it is essential that an individual who is diabetic should take care of dyslipidemia. Our study definitely indicates the presence of hyperlipidemia which might be hazardous for diabetics.

\section{Conclusion:}

The present study suggested that common lipid abnormalities during diabetes induced dyslipidemia are hypercholesterolemia, hypertriglyceridemia and elevated LDL cholesterol. Adequate and proper management of diabetes to reduce dyslipidemia and further complications are therefore recommended.

\section{References:}

1. Ajala MO, Ogunro PS, Idogun SE, Osundeko O. Relationship between plasma antioxidant status and leptin in controlled and non?controlled diabetic non?obese women. International Journal of Endocrinology \&Metabolism. 2009;7(4): 214-221.

2. Choudhury MRS, Alam T, Rahman AKMS. Studies on lipid profile in patients with non insulin dependent diabetes mellitus. KYAMC Journal. 2011; 2(1):123-127.

3. Jain AP, Gupta DP.Study of blood lipid in Diabetics without any manifest vascular complications. Journal Diabetic Association India. 1980; 199: 29-34.

4. Reaven GM: Banting lecture 1988. Role of insulin resistance in human disease. Diabetes. 1988;37(12): 1595-1607.

5. Kaplan NM:The deadly quartet. Upper-body obesity, glucose intolerance, hypertriglyceridaemia and hypertension. Arch Intern Med. 1989; 149(7): 1514-1520.

6. Zimmet P, Alberti G, Shaw J. A new IDF worldwide definition of the metabolic syndrome: the rationale and the results. Diabetes Voice. 2005;50 (3): 31-33.

7. Taskinen MR: Diabetic dyslipidemia. Atheroscler Suppl. 2002;3 (1): 47-51.

8. Folli F, Corradi D, Fanti P, Davalli A, Paez A, Giaccari A et al. The role of oxidative stress in the pathogenesis of type 2 diabetes mellitus micro- and macrovascular complications: avenues for a mechanistic-based therapeutic approach. CurrDiabetes Rev. 2011; 7 (5): 313-24.

9. Maritim AC, Sanders RA, Watkins JB. Diabetes, oxidative stress, and antioxidants: a review. J Bio chem. MolToxicol. 2003; 17 (1): 24-38.

10. Mahato RV, Gyawali P, Raut PP, Regmi P, Khelanand PS, Dipendra RP et al. Association between glycaemic control and serum lipid profile in type 2 diabetic patients: glycatedhaemoglobin as a dual biomarker. Biomed Res. 2011; 22 (3): 375-380.

11. Wexler DJ, Grant RW, Meigs JB, Nathan DM, CaglieroE.Sex disparities in treatment of cardiac risk factors in patients with type 2 diabetes. Diabetes Care. 2005; 28 (3): 514-520.

12. Regmi P, Gyawali P, Shrestha R, Sigdel M, Mehta KD, Majhi S. Pattern of dyslipidemia in type-2 diabetic subjects in Eastern Nepal. J Nepal Assoc Med Lab Sci. 2009;10 (1): 11-13.

13. Onyemelukwe GC, Stafford WL Serum lipids in Nigerians: the effect of diabetes mellitus. Tropical Geographical Medicine 1981; 33: 323-328. 
14. Bello - Sani F, Bakari AG, Anumah FE. Dyslipidaemia in persons with type 2 diabetes mellitus in Kaduna, Nigeria. International Journal Diabetes \& Metabolism 2007; 15: 9-13.

15. Rasha SN. A study of serum lipid profile in obese NDDM patients. Journal Al-Nahrain University.2008. 11 (3):106-110.

16. Anita FP. Clinical Nutrition. 2nd ed. R Dayal, Delhi .1973. 642-646

17. Brownlee M: Biochemistry and molecular cell biology of diabetic complications. Nature. 2001, 414 (6865): 813-820.

18. Jokl R, Colwell JA: Arterial thrombosis and atherosclerosis in diabetes. Diabetes Metab Rev. 1997;5: 1-15.
19. Taskinen MR: Diabetic dyslipidaemia: from basic research to clinical practice. Diabetologia. 2003, 46 (6): 733-49.

20. Goldberg IJ: Diabetic dyslipidemia: causes and consequences. J ClinEndocrMetab. 2001; 8 (3): 965-971.

21. Krauss RM: Lipids and lipoproteins in patients with type 2 diabetes. Diabetes Care. 2004; 27 (6): 1496-1504.

22. Andersen GE, Christiansen JS, Mortensen HB, Christiansen KM, Pedersen-Bjergaard L, Kastrup KW et al. Serum lipids and lipoproteins in 157 insulin dependent diabetic children and adolescents in relation to metabolic regulation, obesity and genetic hyperlipoprotenimia. ActaPaediatr Scand. 1983;72: 361-65. 